\title{
REGIME DE METAS DE INFLAÇÃO DO BRASIL: A INFLUÊNCIA DAS EXPECTATIVAS INFLACIONÁRIAS
}

\author{
Ermeson Henrique Silva dos Reis * \\ Reynaldo Rubem Ferreira Junior ${ }^{\dagger}$ \\ Ariane Danielle Baraúna da Silva $\ddagger$
}

\begin{abstract}
Resumo
O presente trabalho tem por objetivo identificar a influência das flutuações nas expectativas de inflação sobre o Regime de Metas de inflação (RMI), em especial sobre o Índice de Preços ao Consumidor Amplo (IPCA) no Brasil. Destarte, foi possível observar que a expectativa de inflação impacta de maneira positiva a inflação brasileira desde o primeiro mês. No mais, a formação da expectativa futura de inflação é influenciada por um conjunto de variáveis que impactam a inflação de maneiras diferentes tanto em magnitude quando em tempo de duração.
\end{abstract}

Palavras-chave: regime de metas, expectativa de inflação, inflação.

\begin{abstract}
The objective of this study is to identify the influence of fluctuations in inflation expectations on the inflation targeting regime (ITR), in particular on the Extended Consumer Price Index (IPCA). This way, it was possible to observe that inflation expectations have a positive impact on Brazilian inflation since the first month. Beyond this, the formation of the future expectation of inflation is therefore influenced by a set of variables that impact inflation in different ways, both in magnitude and in duration.
\end{abstract}

Keywords: inflation targeting, inflation expectations, inflation.

JEL classification: C81, 121, 128.

DOI: http://dx.doi.org/10.11606/1980-5330/ea148363

\footnotetext{
* Universidade Federal de Alagoas. E-mail: ermesonhenrique@hotmail.com

† Universidade Federal de Alagoas. E-mail: rrfj@uol.com.br

‡ Universidade Federal de Alagoas. E-mail: ariane.danielle.barauna@gmail.com
} 


\section{Introdução}

Na década de 1960 e início de 1970, houve uma clara divisão entre os neokeynesianos e a escola monetarista, que não se deteve apenas à disputa se a "curva IS" ou "Curva LM" era mais ou menos elástica, ou se as políticas monetárias ou as políticas fiscais são mais potentes para a gerência da demanda agregada, que foi apresentada em livros didáticos de graduação, mas se ateve a todo o funcionamento da economia. Já no final dos anos de 1970 e nos anos de 1980 surge a corrente "Novo-Clássica" com a crítica de Lucas. Posteriormente, contrapondo-se criticamente ao pressuposto de Market-clean, ou a não consideração da presença de rigidez de preços e salários no curto prazo, surge a escola dos novos-keynesianos no debate macroeconômico Woodford (2009).

Nesse contexto, torna-se clara a dificuldade de convergência entre as correntes de pensamento econômico no que tange à possibilidade de uma apresentação conjunta de respostas aos problemas econômicos. Contudo, apesar de algumas divergências entre os novos-clássicos, novos-keynesianos e a abordagem dos ciclos reais dos negócios, houve grandes esforços no sentido da construção de um núcleo duro de teorias econômicas capaz de possibilitar maior convergência entre estas escolas, que foi denominado de "novo consenso macroeconômico".

Esse novo consenso sustenta a importância de menor discricionariedade nas ações do Banco Central por meio de um Regime de Metas de Inflação (RMI), no qual o Banco Central teria a missão de coordenar as expectativas dos agentes econômicos em relação à taxa de inflação, usando como instrumento a taxa de juros básica. No mais, existe uma visão difundida, principalmente no Brasil, de que as expectativas de inflação funcionam como um dos mecanismos de transmissão da política monetária. No entanto, identificar e mensurar especificamente o canal das expectativas não tem sido uma tarefa fácil, uma vez que este contamina os demais, como os canais de ativos, crédito, câmbio.

Conforme Bevilaqua et al. (2008), dentro do Regime de metas de inflação as expectativas de inflação têm desempenhado um papel importante no processo de formulação de políticas. Pesquisas regulares sobre como o mercado percebe a inflação fazem parte dos relatórios do Banco Central sendo compilados em um resumo das previsões macroeconômicas no qual são publicadas semanalmente. Para fortalecer incentivos à comunicação precisa de previsões, o Banco Central (BC) publica regularmente classificações dos melhores analistas de curto e médio prazo de várias variáveis. O sistema de pesquisa foi considerado exemplar e foi imitado por outros bancos centrais. De forma geral, as expectativas do mercado quanto à inflação são insumos importantes nos modelos de previsão do BC. Nesse sentido, por meio desses relatórios, tem-se percebido que as expectativas influenciam o comportamento da própria inflação, das metas de inflação, da taxa de câmbio, da evolução dos preços das commodities, da atividade econômica e da orientação da política monetária.

Nesse viés, e com base nos diversos relatórios de inflação, disponibilizado pelo Banco Central do Brasil trimestralmente, a Tabela 1 apresenta a inflação brasileira decomposta em seis componentes: i) variação cambial; ii) inércia associada à parcela da inflação que excedeu a meta; iii) diferença entre expectativas de inflação dos agentes e meta (Exp); iv) choque de oferta (C.oferta); v) inflação de preços livres (IPCA (livres)), excluídos os efeitos dos quatro itens anteriores; e vi) inflação de preços administrados ou monitorados (Pm), retirando-se os efeitos do item "ii)". 
Tabela 1: Decomposição da Inflação Brasileira de 2001 a 2016

\begin{tabular}{lllcccc}
\hline & IPCA & PM & Câmbio & Exp & Inercia & C.oferta \\
\hline 2001 & 31,17 & 22,08 & 37,66 & - & 9,09 & - \\
2002 & 18,4 & 15,2 & 46,4 & 13,6 & 7,2 & - \\
2003 & 11,53 & 18,28 & $-11,83$ & 18,28 & 63,44 & 13,3 \\
2004 & 57,89 & 38,16 & $-3,95$ & 5,26 & 3,95 & 46,3 \\
2005 & 59,65 & 57,89 & $-36,84$ & 5,26 & 14,04 & $-15,5$ \\
2006 & 56,0 & 50,8 & $-17,6$ & $-4,2$ & 14,9 & 5,7 \\
2007 & 112,9 & 21,6 & $-25,1$ & $-9,7$ & 0,3 & 47,5 \\
2008 & 63,9 & 17,8 & 10,7 & 3,7 & 3,9 & 25,8 \\
2009 & 80,5 & 27,3 & $-5,6$ & $-2,2$ & 0,0 & $-5,7$ \\
2010 & 49,9 & 18,5 & $-3,8$ & 3,6 & $-1,6$ & 3,3 \\
2011 & 43,8 & 25,2 & $-34,4$ & 7,8 & 12,0 & 14,5 \\
2012 & 49,7 & 13,5 & 10,2 & 8,3 & 5,8 & 12,5 \\
2013 & 64,3 & 2,5 & 5,9 & 10,7 & 13,4 & 3,2 \\
2014 & 49 & 16,2 & $-0,5$ & 10,8 & 10,9 & 13,6 \\
2015 & 27,8 & 39,5 & 14,7 & 6,8 & 3,1 & 8,1 \\
2016 & 41,7 & 10,2 & $-2,6$ & 10,9 & 29,2 & 10,6 \\
\hline Fonte: Elaborado pelo Autor & & & &
\end{tabular}

Fonte: Elaborado pelo Autor.

De acordo com a Tabela 1, é perceptível inicialmente que as expectativas inflacionárias têm um peso relativamente baixo na inflação brasileira, inclusive tendo em certos anos até peso menor que os choques de oferta, visto que é uma variável em que o Banco Central apenas tenta controlar os efeitos secundários. Por sua vez, observa-se que a inércia inflacionária contribui de maneira substancial, comparando-a com as expectativas, o que leva ao questionamento sobre até que ponto os agentes formam suas expectativas com base na hipótese das expectativas racionais (hipótese básica do regime de metas) e não com base nas expectativas adaptativas. Por isso, a taxa de câmbio, assim como os preços monitorados, representa uma parte significativa da inflação brasileira.

Por sua vez, observa-se que a inércia inflacionária contribui de maneira substancial, comparando-a com as expectativas, o que leva ao questionamento sobre até que ponto os agentes formam suas expectativas com base na hipótese das expectativas racionais (hipótese básica do regime de metas) e não com base nas expectativas adaptativas. Por isso, a taxa de câmbio, assim como os preços monitorados, representa uma parte significativa da inflação brasileira.

Assim, o presente trabalho tem por objetivo identificar a influência das flutuações nas expectativas de inflação sobre o Regime de Metas de Inflação (RMI), em especial sobre do Índice de Preços ao Consumidor - Amplo (IPCA) itens livres no Brasil, durante o período de janeiro de 2001 a setembro de 2017. Para tanto, foram estimadas a curva de Phillips, do lado da oferta, a curva IS, do lado da demanda, assim como a função de reação do Banco Central, que incorpora a regra de política monetária. A estimação foi realizada por meio dos Vetores Autorregressivos Estruturais (SVAR),

O trabalho está dividido em cinco seções além desta introdução. Inicialmente, será apresentado o referencial teórico do trabalho dividido em dois subtópicos: o primeiro subtópico estabelecendo a estrutura do novo consenso e o segundo subtópico apresentando os canais de transmissão da política monetária e os seus efeitos sobre a atividade econômica. Na sequência, serão expostos os fundamentos metodológicos, as análises de resultados e algumas 
conclusões, respectivamente.

\section{Novo Consenso Macroeconômico}

Apesar das divergências entre as principais escolas do mainstream macroeconômico, há evidências de esforços no sentido da construção de um núcleo duro de teorias econômicas capazes de possibilitar maiores convergências entre essas escolas, que tem início na década de 1990. Esse Novo Consenso Macroeconômico (NCM) tem por princípio que a prioridade da política monetária é a estabilidade de preços utilizando como instrumento a taxa de juros. É válido lembrar que um dos aspectos mais importantes desse novo consenso é a maior aproximação entre a academia e os formuladores da política monetária (policymarker). Nessa perspectiva, os Bancos Centrais devem gerir a política monetária tendo como principal mandato a estabilidade de preços.

Em resumo, o novo consenso fundamenta-se em um modelo estrutural baseado em três equações: a) uma curva de demanda agregada; b) uma curva de oferta agregada; e c) uma regra de política monetária. De acordo com esse modelo, o principal instrumento que o Banco Central detém é a taxa de juros para correção do excesso (ou insuficiência) de demanda e convergência das expectativas de inflação em direção à meta. A estrutura aqui apontada está baseada na versão apresenta por Clarida et al. (1999) por captar a essência no novo consenso. Trata-se, em linhas gerais, de um modelo dinâmico de equilíbrio geral com rigidez temporária de preços nominais, estruturado no modelo novo keynesiano de expectativas racionais. A diferença existente entre o modelo IS-LM é que as equações agregadas comportamentais evoluem a partir da otimização das firmas e dos indivíduos (houve uma tentativa de microfundamentar o modelo), além disso, o modelo tem por base que o comportamento da economia depende das expectativas em relação ao curso futuro da política monetária (as expectativas são endógenas ao modelo diferente do modelo ISLM).

A equação (1) representa o hiato do produto, sendo que o hiato é dado pelo distúrbio entre produto corrente $y_{t}$ em relação ao produto potencial $z_{t}$, que é o produto onde não há rigidez de preços, isto é, o produto de longo prazo onde a economia se encontra ao nível da taxa natural de desemprego. Tanto o produto do período $t$ quanto o produto potencial são componentes estocásticos com distribuição de probabilidade conhecida.

$$
x_{t}=y_{t}-z_{t}
$$

Com essa definição em mente, é possível apresentar a curva de IS e a curva de Phillips, como se segue:

$$
\begin{gathered}
x_{t}=\theta_{1} E_{t} x_{t+1}-\theta_{2}\left[i_{t}-E_{t} \pi_{t+1}\right]+g_{t} \\
\pi_{t}=\beta_{1} x_{t}+\beta_{2} E_{t} \pi_{t+1}+u_{t}
\end{gathered}
$$

Aqui, $i_{t}$ é a taxa de juros nominal no período, a taxa de inflação no período $\pi_{t}$ é a expectativa de inflação para o período $E_{t} \pi_{t+1}$ e, por fim, é a expectativa do hiato do produto no período $E_{t} x_{t+1}$. Tem-se ainda que $g_{t} u_{t}$ são os termos de erros que atendem respectivamente. Os erros são variáveis identicamente distribuídos com média zero e variância constante. 
Na equação (1), que representa a curva de IS, é possível observar que a curva sofre a influência do produto esperado e da taxa real de juros, diferentemente da curva IS tradicional, de maneira que, se a taxa de juros nominal estiver acima da expectativa de inflação futura, isso indica que a taxa real de juros é positiva. Com isso, haverá uma diminuição do hiato do produto, uma vez que ocorre intertemporalmente a substituição de consumo presente por futuro, dada pela elasticidade captada por $\theta$. Outro fator importante é que o produto corrente sofre a influência positiva do hiato do produto futuro, já que, quando se espera um crescimento do produto, no futuro haverá um crescimento do consumo e no produto corrente. Por fim, o termo de erro representa um choque de demanda capaz de deslocar a curva de IS, como é o caso de um aumento dos gastos do governo.

Com relação à curva de Phillips (3), essa é derivada a partir do processo de determinação de preços das firmas individuais. Diferentemente da curva tradicional, a inflação depende tanto da expectativa quanto das condições econômicas correntes e futuras, como também da expectativa de inflação futura. Isso indica que a inflação sofre interferência de como a economia vai se comportar no futuro e também como os polycymakers estão operando.

A terceira função que fundamenta o novo consenso macroeconômico é uma regra de taxa de juros, que pode ser uma trajetória futura exógena de taxas de juros nominais ou reais, ou uma regra de reação do tipo Taylor (com pesos para os desvios da inflação esperada em relação à meta), ou ainda uma regra de reação ótima, calculada determinística ou estocasticamente Bogdanski et al. (2000).

Essa representação de política monetária é derivada a partir da curva de reação do Banco Central ou regra de Taylor.

$$
i_{t}=\alpha+\gamma_{\pi}\left(E_{t} \pi_{t+1}-\bar{\pi}\right)+\gamma_{x} x_{t}
$$

Aqui, $i_{t}$ trata-se da taxa de juros nominal, $\bar{\pi}$ é a meta de inflação seguida pelo Banco Central e, como visto, representa o hiato do produto. Observa-se que, quando a expectativa de inflação está acima da meta, o Banco Central reagirá elevando a taxa nominal de juros, para que posteriormente o hiato do produto caia (equação (4), fazendo com que a inflação volte para o centro da meta. Todavia, quanto à curva de Reação, o Banco Central também estabelece uma relação com o nível de produto, sendo que se o mesmo estiver acima do potencial, então, a autoridade monetária reage, de modo a elevar a taxa de juros para conter possíveis pressões inflacionárias. O contrário também é válido, quando a inflação está abaixo da meta e o produto abaixo do potencial, o Banco Central reduz a taxa de juros.

Arestis \& Sawyer (2008) trazem que os elementos-chave do modelo de metas de inflação podem ser resumidos da seguinte forma: (i) um quadro de política monetária em que o anúncio público de metas de inflação, ou intervalosalvo, é realizado com o reconhecimento explícito de que a estabilidade de preços é o objetivo primordial da política monetária; (ii) a taxa de juros é tida como o principal instrumento da política macroeconômica; (iii) a existência de um Banco Central "independente" é essencial para a boa atuação da política monetária; (iv) a política monetária apenas diz respeito à taxa de inflação e seus possíveis efeitos. 


\section{Regime de Metas de Inflação do Brasil}

O sistema ou regime de metas para a inflação (RMI), conceitualmente, "é um regime monetário no qual o Banco Central se compromete a atuar de forma a garantir que a inflação efetiva esteja em linha com uma meta pré-estabelecida, anunciada publicamente" (BCB - DIPEC, 2010, p.7). O RMI é instituído oficialmente no Decreto Presidencial no 3.088, de 21 de junho de 1999, em que a taxa de juros (SELIC) se constitui na variável-operacional para alcançar determinada meta de inflação. Em 30 de junho de 1999, o Conselho Monetário Nacional (CMN) edita a Resolução $n^{\circ} 2.615$, obtendo a definição do índice de preços de referência e das metas para a inflação de 1999 e para o ano subsequente. Houve, com o passar do tempo, algumas mudanças na estrutura do regime de metas no Brasil, principalmente nas curvas de oferta e demanda. A principal inovação está no aprimoramento de cenários de política econômica, relacionando de maneira direta o swap pré-DI com a trajetória da taxa Selic. Além disso, buscou-se incorporar impactos de choques de oferta na Curva de Phillips, condicionada à percepção da importância dessa variável na inflação brasileira. A estrutura das equações expostas aqui está baseada no relatório do Banco Central do Brasil (BACEN) (2014), que serve de base para esse trabalho. Com isso, tem-se:

$$
\begin{gathered}
\pi_{t}^{L}=\sum_{i>0} a_{1, t} E_{t} \pi_{t+i}+\sum_{j>0} a_{2, j} \pi_{t-j}+\sum_{k>0} a_{3, k} c b r_{t-k} \\
+\sum_{l>0} a_{4, l} h_{t-j}+\sum_{m>0} \sum_{n>0} a_{5, m}^{n} Z_{t-m}^{\pi, n}+\epsilon_{t}
\end{gathered}
$$

$\mathrm{O} \pi_{t}^{L}$ trata-se da inflação de preços livres do IPCA, $E_{t} \pi_{t+i}$ é a expectativa corrente acerca da inflação $i$ trimestres à frente, $\pi$ é a inflação plena medida pela variação do IPCA, $c b r_{t}$ é a inflação externa medida pela variação do índice Commodity Research Bureau (CRB) expresso em moeda nacional, $h_{t}$ tratase de uma medida do hiato do produto, $Z_{t}^{\pi, n}$, a variável de controle $n, \epsilon_{t}$ é um termo de erro. As variáveis de controle são proxies para choques de oferta, por exemplo, flutuações no preço internacional do petróleo (em reais) e o descasamento entre preços no atacado e no varejo.

A curva IS demonstra a dinâmica do hiato do produto como função das suas defasagens, da taxa real de juros, de variáveis fiscais, externas e do mercado de crédito. Assim:

$$
\begin{aligned}
h_{t}=\beta_{0} & +\sum_{i>0} \beta_{1, i} h_{t-i}+\sum_{j>0} \beta_{2, j} r_{t-j}+\sum_{k>0} \beta_{3, k} \Delta s u p_{t-k} \\
& +\sum_{l>0} \beta_{4, l}\left(\Delta h_{t-j}^{*}+\Delta \bar{y}_{t-l}^{*}+\Delta \bar{y}_{t-l}\right)+W_{t}+u_{t}
\end{aligned}
$$

Nesta, $r_{t}$ é a taxa de juros medida pela taxa de juros nominal swap pré-DI de 360 dias deflacionada pela expectativa de inflação relativa ao período de vigência do contrato de swap; $\Delta s u p_{t}$ é a variação do superávit primário estrutural, $\bar{y}_{t}^{*}$ é a taxa de crescimento do produto mundial; $\Delta h_{t}^{*}$ é a variação do hiato do produto mundial; $\Delta \bar{y}_{t}^{*}$ é o crescimento do produto potencial mundial; $\Delta \bar{y}_{t}$ é o crescimento do produto potencial doméstico; $W_{t}=\sum_{n>0} \sum_{p>0} \beta_{5, n}^{p} Z_{t-n}^{h, p}$ 
é variável de controle, na qual pode-se incluir, entre outras, variáveis indicadoras de condições financeiras, de grau de confiança dos agentes, de grau de incerteza e de condições de crédito.

No que diz respeito à função de reação do Bacen para a condução da política monetária, ela é bastante similar à curva de Taylor apresentada por Bogdanski et al. (2000), uma vez que não é apresentado em nenhum relatório que a regra passou por qualquer tipo de modificação. Ademais, nos relatórios de setembro de 2010 e julho de 2011, é apresentada a regra de Taylor como constituída pela taxa de juros doméstica (Selic) em função do hiato do produto e do desvio da inflação em relação a sua meta.

\subsection{Estimações da Curva de Phillips, IS e da Regra de Taylor no Brasil}

As estimações das curvas referentes ao RMI fazem parte da discussão atual da macroeconomia. Muitos trabalhos buscaram estimar as curvas de oferta e demanda, assim como a função de reação do Banco Central com modelos cada vez mais sofisticados. No Brasil, da mesma forma como tem acontecido no mundo, vários trabalhos acadêmicos procuraram realizar estimações, por meio das premissas estabelecidas do Novo Consenso Macroeconômico, e aqui serão listados alguns desses trabalhos em relação às curvas do RMI.

Com relação às estimações da Curva de Phillips feitas no Brasil por Minella et al. (2002), que tem por objetivo avaliar a política monetária do Banco Central, observou-se que a inflação tem funcionado como um importante indicador de expectativas futuras de inflação e que o Banco Central tem reagido de maneira forte em relação às expectativas de inflação. Pode-se ressaltar também outro ponto importante no trabalho, que é a passagem da taxa de câmbio para os preços "administrados ou monitorados", sendo duas vezes maior do que para os preços de "mercado". Optando por uma estimação um pouco diferente, Areosa (2004) estima a Curva de Phillips Neokeynesiana padrão (CPNK) e a Curva Híbrida de Gali e Gertler (1999) com base no método generalizado dos momentos (MGM).

Os resultados podem ser resumidos da seguinte forma: (i) o Brasil apresentou uma maior rigidez nominal do que os Estados Unidos e Europa; (ii) com relação à economia aberta com indexação, uma desvalorização do câmbio nominal acrescido da inflação externa interfere na inflação ao consumidor; (iii) o impacto direto das variáveis relacionadas à abertura econômica é pequeno, sendo o somatório de seus coeficientes próximo a zero; e (iv) por fim, o impacto indireto é significativo, o que altera de maneira consistente os pesos associados à inflação defasada e à expectativa da inflação futura.

Tentando entender melhor o impacto do câmbio na inflação, Correa \& Minella (2005) estimam uma curva de Phillips com limiar (threshold) para o repasse cambial na tentativa de identificar a presença de mecanismos não lineares de repasse cambial na inflação no Brasil. Os resultados indicam que o repasse de curto prazo é menor quando a economia está em depressão, quando a taxa de câmbio se deprecia acima de certo valor e quando a volatilidade da taxa de câmbio é menor.

Nessa linha, Carneiro et al. (2002) realizaram estimativas da curva de Phillips para avaliar a não linearidade do repasse cambial para inflação. Nesse processo, foram estimadas uma Curva de Phillips Backward-Looking com coeficiente de repasse constante e uma Curva de Phillips Backward-Looking com um coeficiente de repasse não linear, em função da taxa de câmbio real e da 
taxa de desemprego dessazonalizada. Essa especificação é realizada pelo Modelo não linear. Os resultados demostram a existência de diferentes mecanismos não lineares de repasse da variação cambial entre os diferentes grupos de produtos e serviços que compõem a cesta do IPCA. Logo, a relação entre a inflação e o câmbio não pode ser apresentada apenas pela especificação linear.

Em uma análise com dados mensais, Schettini et al. (2012) estimam uma curva de Phillips por meio do modelo Vetorial Autorregressivo (VAR). Entre os resultados econométricos, observa-se: a) o impacto de um choque cambial na inflação é de aproximadamente 0,04 pontos percentual (p.p.) b) um choque médio na taxa de desemprego demora em média 18 meses para desaparecer; c) a resposta da inflação é de 0,049 p.p. a choques nas expectativas de inflação e d) choques na série de inflação não afetam a taxa de desemprego, ou seja, inflação maior não afeta a taxa de desemprego.

Já Carrara (2016), por meio de uma estimação com o VAR e o VEC, expõe que na curva de Phillips a expectativa de inflação impacta de maneira direta e rápida inflação. Além disso, os choques de oferta relacionados ao impacto do $\mathrm{CRB}$, os choques de câmbio e os choques de produtividade têm impacto significativo, embora não seja um impacto imediato. Por fim, o hiato do produto não se mostrou relevante para explicar as flutuações da inflação.

Em relação à estimação da curva IS no Brasil, a quantidade de trabalho não é tão abrangente quando comparado à curva de Phillips. Mesmo assim, surgiram alguns trabalhos que ajudarão no entendimento da pesquisa, entre eles, Freitas et al. (2001) estimam uma curva IS pós-plano real na busca de determinar os mecanismos da política monetária. O método utilizado na estimação foi o OLS. Os resultados da curva IS foram que os coeficientes da taxa de juros e do hiato do produto são muito significativos e com o sinal esperado, e que, em relação à taxa de juros, a expectativa da demanda agregada se reduz a $0,39 \%$.

Em conformidade com o trabalho acima, Bonomo \& Brito (2002) buscaram identificar as políticas monetárias ótimas dentro das dinâmicas de curto prazo da economia brasileira. Essa modelagem foi realizada por meio de uma estimação que simula um modelo macroeconômico aberto sobre a premissa das expectativas racionais. Os autores chegaram à conclusão de que a taxa de juros tem efeito significativo sobre o hiato do produto, assim como Andrade \& Divino (2001). Contudo, a taxa de câmbio não se mostra significativa, além de o coeficiente ter o sinal inverso ao esperado.

Entretanto, Santos \& Holland (2008) estimaram uma curva IS para a economia brasileira pelo modelo VAR utilizando algumas variações nos modelos em relação à taxa de juros ex-ante ou ex-post. Em suma, os resultados podem ser resumidos da seguinte forma: a) as mudanças na taxa real de câmbio não representam um papel significativo na demanda agregada doméstica, os termos de troca mostram-se estatisticamente significantes, b) e o impacto do crescimento econômico global foi maior sobre a economia brasileira do que em relação a outras economias.

Em contraponto aos autores acima, Carrara (2016) propõe uma estimação da curva IS a partir do VEC, utilizando uma curva baseada no modelo de médio porte utilizado pelo Banco Central. Os resultados do trabalho mostram que a taxa de juros é o principal mecanismo que interfere no hiato do produto, o que traz evidência para o fato de que a taxa de juros pode ser um instrumento eficaz de política monetária. Pode-se destacar ainda que a taxa 
de câmbio e as expectativas de inflação contribuem para determinar as flutuações da demanda, algo condizente com a teoria.

Em relação à curva de reação do Banco Central, vários trabalhos foram produzidos a partir da adoção do RMI. Nesse contexto, Minella et al. (2002) estimam funções de reação para o Banco Central do Brasil (BACEN) do tipo forward-looking. De maneira geral, os autores observam que o BACEN responde fortemente aos desvios entre a expectativa de inflação e a meta. Nesse contexto, Souza Júnior \& Caetano (2014) estimaram a Regra de Taylor com o objetivo de avaliar a relevância das decisões de política monetária para o caso de uma função de produção tipo Cobb Douglas, que é mais complexa que o uso do filtro HP. Para o processo de estimação, utilizou-se o Método Generalizado dos Momentos - Generalized Method of Moments (GMM). Os resultados revelaram que a função de produção Cobb-Douglas pode ser uma ferramenta interessante para a estimação da curva de Taylor e que o BCB tem optado por uma política mais lenta em relação às mudanças da economia.

Soares \& Barbosa (2006), por sua vez, estimaram uma curva de Taylor no Brasil a partir da implementação do sistema de metas de inflação em junho de 1999. O ponto diferencial desse trabalho é que se utilizou muito a taxa de juros real de equilíbrio de longo prazo em relação à meta de inflação variável no tempo. De acordo com os resultados, existe uma evidência empírica de que a política monetária segue a regra de Taylor desde que se inclua a taxa de câmbio real corrente e defasada. Sendo que, de maneira geral, observouse uma boa robustez no modelo no qual os coeficientes se comportaram da maneira esperada.

Em uma estimação bastante clássica, Dezordi et al. (2009) realizam uma estimação do regime de metas de inflação com base em uma curva de Taylor expondo a relação entre a taxa de juros e o hiato do produto e a inflação, a estimação é realiza por meio do VAR. Os resultados apontaram que, no caso da economia brasileira, é possível observar que a mesma pode ser orientada pela regra de Taylor. Além disso, na função de reação do Banco Central, um choque de $1 \%$ na inflação leva o Banco Central a aumentar a taxa Selic em $1,9 \%$, já em relação ao hiato do produto, a taxa de juros deve elevar-se em 0,98\% no acumulado. Em geral, os resultados revelaram que o Banco Central é mais preocupado com a inflação do que com o crescimento da economia.

Por fim, destaca-se o trabalho de Mendonça et al. (2005), que, por meio do VAR, estimam uma regra de Taylor com as seguintes variáveis: i) Taxa de juros nominais ii) Taxa de juros real iii) Hiato do produto iv) Inflação v) Risco País. Observou-se que a taxa de juros no Brasil não tem sido utilizada de maneira correta e que os sucessivos descumprimentos das metas de inflação dificultam a redução da taxa de juros pelo Banco Central, sem comprometer as expectativas dos agentes econômicos com o descontrole da inflação. Assim, a taxa de juros pode não ser um bom instrumento de combate à inflação, podendo levar a resultados inversos ao esperado. Um ponto importante a se destacar é que, embora a taxa de juros não responda à inflação e ao hiato do produto, a mesma responde aos choques do índice de risco país.

O presente trabalho possui como ponto diferencial, frente a todos os expostos aqui, a tentativa de identificar o papel que as expectativas têm no regime de metas, algo pouco evidenciado até então. Nas estimações das curvas estabelecidas aqui, será sempre exposta a possibilidade de identificar como a expectativa reage a possíveis distúrbios dos componentes tanto da curva de oferta como da curva de demanda. 
O intuito dessa análise é demostrar não só a relação entre expectativas e inflação, mas identificar as variáveis que mais afetam a expectativa gerando efeitos mais contundentes sobre a inflação, tais como: câmbio, gastos governamentais, índice de commodities, etc. conforme é demonstrado nos resultados. Dessa forma, a política monetária de controle das expectativas de inflação pode ter algumas limitações, uma vez que as mudanças na expectativa de inflação podem estar, em parte, relacionadas a fatores que estão fora do campo de atuação do BC.

\section{Metodologia}

\subsection{Modelo Empírico}

Levando-se em consideração a discussão teórica a respeito do papel que as expectativas têm na inflação brasileira, apresentam-se a seguir as equações de Phillips, curva IS e a função de reação do BACEN.

$$
\begin{gathered}
\pi_{t}=\alpha \pi_{t-j}+\beta p m_{t-j}+\gamma \exp _{t+j}+\delta e_{t-j}+\varphi h_{t-j}+\phi i c b r_{t-j}+\epsilon_{1, t} \\
h_{t}=\theta h_{t-j}+\mu r_{t-j}+\xi e_{t-j}+n f s p_{t-j}+\lambda \exp _{t+j}+\epsilon_{2, t} \\
i_{t}=\sigma i_{t-j}+\phi D_{t-j}{ }^{1}+\rho h_{t-j}+\epsilon_{3, t}
\end{gathered}
$$

A descrição das variáveis está na Tabela 2 abaixo, assim, existem algumas divergências dessa expressão com a Curva de Phillips tradicional do Banco Central. Inicialmente, optou-se por analisar a inflação de preços livres e administrados de maneira separada, para identificar os possíveis efeitos de propagação entre as variáveis. Em segundo lugar, atribui-se à Curva de Phillips uma proxi que representa o choque de oferta (icbr), o qual contempla a parte da inflação que não responde diretamente à política monetária. Assim buscase analisar até que ponto a inflação brasileira é derivada de fatores ligado à demanda e pelo canal das expectativas. Por fim, o o hiato do produto é calculado conforme o filtro Hodrick-Prescott.

Tabela 2: Descrição das Variáveis Utilizadas no Modelo

\begin{tabular}{cl}
\hline & Descrição das Variáveis \\
\hline$\pi$ & Taxa de Inflação de Preços Livres \\
$\mathrm{pm}$ & Preços Monitorados \\
$\exp$ & Expectativa de Inflação \\
$\mathrm{e}$ & Taxa de Câmbio \\
$\mathrm{h}$ & Hiato do Produto \\
$\mathrm{cbr}$ & Índice de Commodities do Brasil \\
$\mathrm{r}$ & Taxa Referencial - swaps - DI pré-fixada - 360 dias \\
$\mathrm{nfsp}$ & Necessidade de Financiamento para Superávit Primário \\
$\mathrm{i}$ & Taxa Selic Efetiva \\
$\mathrm{D}$ & Desvio das Expectativas em Torno da Meta \\
\hline
\end{tabular}

Fonte: Elaborada pelo Autor.

\footnotetext{
${ }^{1}$ No cálculo do desvio da expectativa de inflação em torno da meta, optou-se por realizar o método proposto por Minella et al. (2002)
} 
É importante salientar que na curva de IS introduzimos a variável expectattiva na busca de demonstrar qual o grau de perturbação da demanda da economia (perturbação pela qual o Banco Central busca conter), dados os choques de expectativa. Com isso, identificar-se-á o quanto o hiato do produto se modifica em relação às flutuações das expectativas dos agentes em relação à inflação. Essa percepção é importante, já que o Banco Central busca controlar as perturbações de demanda via controle de expectativas do mercado em relação à inflação futura.

Para a estimação da Curva de Phillips e da Regra de Taylor, serão utilizados dados mensais que vão de janeiro de 2001 setembro de 2017. Para a curva IS, foram utilizados dados mensais que vão de janeiro de 2002 a setembro de 2017.

\section{Estimativa e identificação}

\subsection{Modelo}

A estrutura do Modelo SVAR tem a seguinte estrutura geral:

$$
y_{t}^{\prime} A_{0}=\sum_{l=1}^{v} A_{l}+c+\epsilon_{t}^{\prime}
$$

onde $y_{t}$ é um vetor $n \times 1$ de variáveis endógenas, $\epsilon_{t}$ é um vetor $n \times 1$ de choques estruturais, A é uma matriz $n \times n$ de parâmetros estruturais para $0 \leq l \leq v$ e com $A_{0}$ invertível. $c$ é um vetor $1 x n$ de parâmetros, $v$ é o número de defasagem e $T$ é o tamanho da amostra. O vetor $\epsilon_{t}$ depende das informações passadas e das condições iniciais $y_{0}, \cdots, y_{1-v}$, é gaussiano com zero médio e matriz de covariância $I_{n}$ (a matriz de identidade $n \times n$ ). O SVAR descrito na equação (10) pode ser escrito como

$$
y_{t}^{\prime} A_{0}=x_{t}^{\prime} A_{+}+\epsilon_{t}^{\prime}
$$

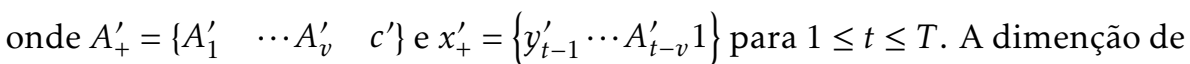
$A_{+}$é $m x n$ onde $m=n v+1 . A_{0}$ and $A_{+}$são os parâmetros estruturais. A forma reduzida dos vetores autorregressivos (VAR) implica a seguinte equação:

$$
y_{t}^{\prime}=x_{t}^{\prime} B_{+}+u_{t}^{\prime}
$$

onde $B=A_{+} A_{0}^{-1}, u_{t}^{\prime}=\epsilon^{\prime} A_{0}^{-1}$ e $E\left[u_{t} u_{t}^{\prime}\right]=\sum=\left(A_{0} A_{0}^{\prime}\right)^{-1}$.

É sabido que, uma vez que os parâmetros estruturais não são identificados, para recuperar os choques estruturais, precisamos impor algumas restrições de identificação. Como vemos a seguir, todos os nossos esquemas de identificação restringem apenas na identificação do choques de expectativas. Como identificamos apenas um choque, o SVAR é parcialmente identificado. Além disso, impomos menos que $n-1$ restrições a zero. Assim, como mostra RubioRamirez et al. (2010), o SVAR é definido identificado independentemente do número de restrições de sinal impostas.

\subsection{Identificação}

A Tabela 3 apresenta os resultados da aplicação do teste Dickey-Fuller aumentado (ADF), o Phillips-Perron (PP). Em relação às variáveis da curva de 
Phillips, observa-se que a inflação de preços livres (IPCA (livres)), a inflação de preços monitorados (PM), o hiato do produto (hiato) e as expectativas inflacionárias (exp) são séries estacionárias em nível ou I(0), em todos os testes. Já o IC-br e o câmbio apresentam uma raiz unitária, logo, não são estacionárias ou I(1).

No mais, as variáveis NFSP, juros DI da curva IS, também não são estacionárias em nível, apenas na sua primeira diferença ou I(1). Com relação às variáveis apenas pertencentes à curva de reação do Banco Central, observa-se também que tanto a Selic quanto o desvio das expectativas em torno da meta não são estacionárias em nível.

Tabela 3: Testes de Estacionaridade ADL e PP

\begin{tabular}{lcc}
\hline & ADL & PP \\
\hline IPCA (livres) & P-valor & P-valor \\
Pm & 0,000 & 0,000 \\
Câmbio & 0,944 & 0,000 \\
Exp & 0,035 \\
Hiato & 0,000 & 0,000 \\
IC-br & 0,324 & 0,000 \\
Juros-DI & 0,836 & 0,612 \\
NFSP & 0,000 & 0,000 \\
Selic & 0,925 & 0,587 \\
Desvio & 0,840 & 0,242 \\
\hline
\end{tabular}

Fonte: Elaborada pelo Autor.

Na curva de Phillip, foram colocadas as seguintes restrições: (i) as expectativas sofrem influência em relação à inflação de preços livres e administrados como também do câmbio e do desvio do produto em relação ao potencial; (ii) e a segunda restrição impõe que essa relação é positiva, uma vez que, quando há uma expansão das variáveis da curva de Philips, o consumidor reage aumentando suas expectativas de inflação futura. Tendo por base que esses efeitos decorrentes de a um excesso de demanda. Já na Curva IS, as expectativas também têm uma relação positiva com o hiato do produto, câmbio e taxa de juros nominal. No caso da necessidade de financiamento, impomos que o efeito é zero.

A identificação da regra de política monetária é motivada pelas regras do tipo Taylor amplamente usadas nos modelos DSGE, dentro da literatura SVAR, no qual assumem que a autoridade monetária reage às mudanças na atividade econômica e nos preços. Logo assumimos que: (i) taxa Selic é o instrumento de política monetária e só reage de maneira contemporânea ao produto e aos desvios das expectativas em relação à média; (ii) a reação da taxa Selic à atividade econômica e ao desvio das expectativas em relação à meta de inflação é positiva. É importante salientar que quando os bancos centrais definem a taxa básica de juros, eles não têm dados sobre produtos e preços disponíveis para o mês atual. No entanto, os bancos centrais têm acesso a uma enorme quantidade de indicadores em tempo real (relatórios semanais de emprego, pesquisas de negócios, dados do mercado financeiro) para aprender sobre o estado atual das atividades e preços reais. 
Por fim, como os dados são mensais, utilizou-se o número de defasagem igual a 12 meses conforme é amplamente utilizado na literatura.

\section{Resultados}

\subsection{Curva de Phillips}

Nessa subseção, serão apresentadas as funções impulso resposta, como parte fundamental do trabalho. Assim, a figura 1, apresenta as respostas das variáveis da curva de Phillipsa a impulsos de expectativas. O objetivo aqui é demostrar o impacto da expectativa e das demais variáveis sobre a inflação de preços livres.

É interessante ressaltar que a diferença obtida aqui neste trabalho em relação às demais estimações expostas acima parece estar apenas na magnitude da interferência da expectativa sobre a inflação. A estimação demonstrada na figura 1 apresenta uma resposta do IPCA (livres) menor do que a que foi apresentada nos demais trabalhos, essa diferença de resultado pode estar condicionada às peculiaridades da função estimada. Uma dessas diferenças utilizadas neste trabalho é no desmembramento do IPCA (amplo) em preços livres (IPCA (livres)) e preços monitorados (PM), com isso um choque de expectativa é direcionado apenas para a inflação de preços livres, em vez do índice cheio.

Logo, percebe-se que a inflação brasileira é, em parte, influenciada pelos choques de expectativa da própria inflação e que a construção de uma curva de Phillips forward-looking pode ser coerente para a economia brasileira. Um resultado similar foi encontrado no trabalho de Schettini et al. (2012). Com isso, pode-se afirmar que quando o Banco Central consegue interferir na expectativa inflacionária, essa interferência é repassada para a inflação, constituindo, assim, uma das formas pelo qual o Banco Central consegue interferir na inflação.

Colaborando com essa assertiva, Vicente \& Graminho (2015) demostram que a expectativa de inflação é o principal componente da inflação implícita. Logo, é possível ter informações valiosas sobre a inflação futura analisando a diferença entre as taxas de juros nominal e real.

Outro autor que encontrou um resultado parecido foi Carrara (2016), em que a resposta do IPCA a um choque de expectativa é de quase um para um. Assim, um choque de expectativa é quase totalmente repassado para a inflação, evidenciando o argumento de Carroll (2003) para quem a expectativa de inflação e a taxa de desemprego corroboram para a explicação das mudanças inflacionárias.

Além desses autores, Correa \& Minella (2005) demonstram, em uma estimação diferente da que foi proposta nesse trabalho, que a expectativa de inflação tem uma influência maior do que a própria inércia, ou seja, a expectativa de inflação tem maior influência sobre a inflação do que a inflação passada.

No mais, é importante destacar que as expectativas inflacionárias também influenciam outras variáveis da curva de Philips. Perceba que um choque de expectativas gera um efeito de desvalorização cambial significativo até o 4 mês. Com isso, o fato de o câmbio ser influenciado pela a expectativa revela a importância desse choque no canal de transmissão do câmbio, sendo que além da pressão direta que o câmbio sofre sobre a inflação de preços via passthrough, existe também o efeito do câmbio sobre a inflação preços por meio das expectativas. Observe que as expectativas também geram pressão para o 
aumento dos preços monitorados e para o índice de commodities do Brasil, gerando também efeitos indiretos na inflação tanto via choque de oferta quando no reajuste das tarifas dos preços monitorados.

Figura 1: Respostas das Variáveis da Curva de Phillips à Choques de Expectativas
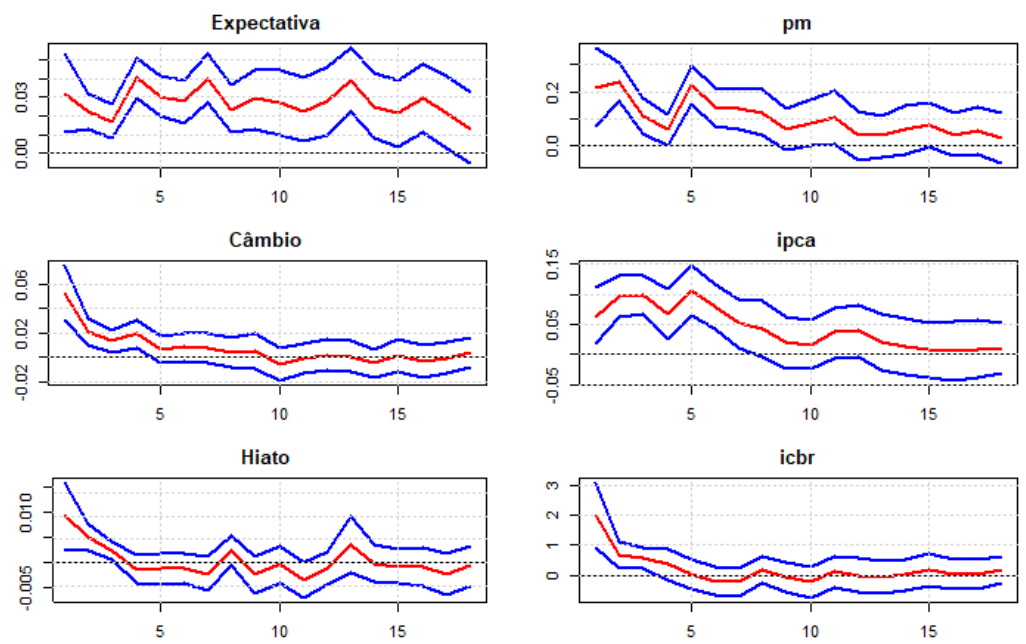

Fonte: Elaborado pelo Autor.

Existe um elemento importante a ser ressaltado aqui, no qual tem sido pouco explorado pelos demais trabalhos que buscaram estimar a curva de Phillips. Com base nessa modelagem, que buscou se aproximar da metodologia de decomposição da inflação pelo BACEN, ficou evidente que embora o impulso na expectativa de inflação gere mudanças na inflação de preços livres, esta sofre influência significativa de fatores ligados à oferta da economia, o que pode gerar um ambiente de certa indexação. Esse resultado pode demonstrar que a capacidade de atuação do BACEN de conter os efeitos inflacionários pode ter limitações, uma vez que a atuação do Banco Central via canal das expectativas, assim como os demais, busca conter primariamente a inflação de demanda.

Após essa estimação, é importante entender que o impacto das expectativas na inflação não é fácil de ser percebido, uma vez que a expectativa inflacionária está diretamente interrelacionada com todas as variáveis do sistema. Assim, na tentativa de determinar quais os fatores que levam a mudanças na expectativa de inflação, fato pouco explorado pelos trabalhos que buscaram estimar a curva de Phillips.

\subsection{Curva IS}

Já as respostas da demanda agregada aos choques de expectativas é apresentado nesta secção conforme a figura 2. Verifica-se, inicialmente, que um aumento das expectativas de inflação faz como que o hiato do produto tenha uma tendência de crescimento no primeiro mês e logo após uma queda que se estende até o $3^{\circ}$ mês no qual o resultado passa a não ser significativo. Entretanto, esse aumento é pequeno tanto em magnitude quanto em tempo de duração, resultando na possibilidade de que o efeito de propagação de um 
choque de expectativa não gere efeitos contundentes na inflação via mudança na demanda agregada.

Assim, sabendo que o hiato do produto interfere na inflação e como a expectativa de inflação não tem um efeito expressivo sobre a demanda agregada, a possibilidade de o Banco Central intervir na economia via canal das expectativas fica limitada, uma vez que, quando o Banco Central sinaliza uma mudança na taxa de juros futura, esse anúncio não levará a mudanças gradativas na demanda e, por sua vez, na inflação.

Figura 2: Respostas das Variáveis da Curva de IS à Choques de Expectativas
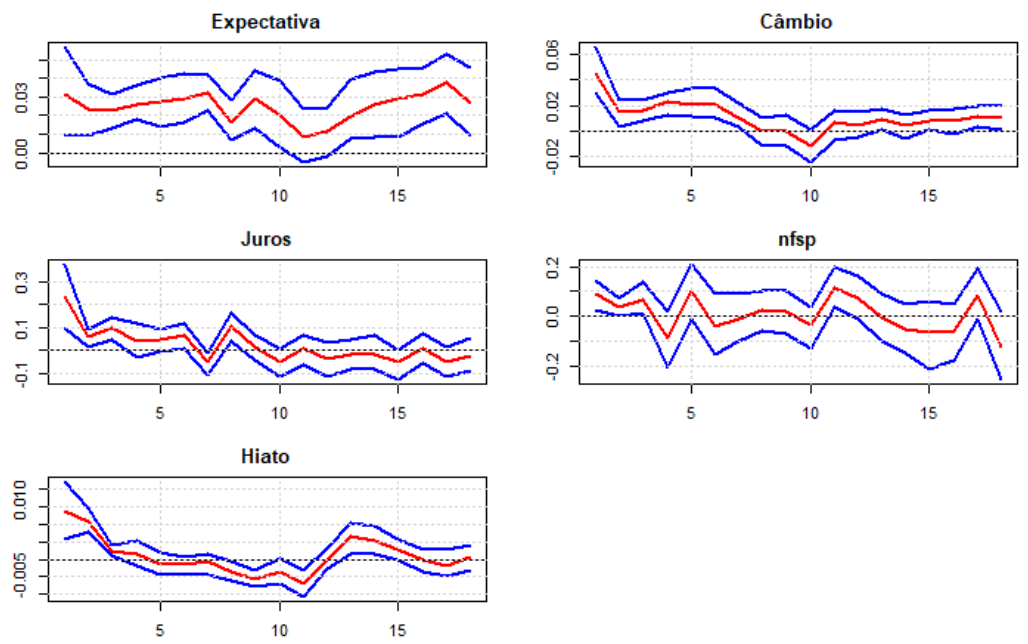

Fonte: Elaborado pelo Autor.

Já a figura 2 mostra os aspectos que interferem na expectativa pelo lado da demanda, que podem, assim, influenciar a inflação pelo canal das expectativas ou pelos outros canais, dadas as suas inter-relações. O objetivo é determinar as fontes que geram aumento da expectativa inflacionária, uma vez que esse aumento levará em um segundo momento a perturbações da inflação.

A resposta da taxa de juros DI na expectativa também é exposto na figura 2. Um choque na expectativa de inflação faz com que a taxa de juros aumente e tenha, posteriormente, uma tendência de queda, embora passasse por um período inicial de instabilidade. Esse fato está condicionado à percepção dos agentes de que a inflação futura cairá devido à política monetária restritiva.

O resultado reforça a premissa de que a taxa de juros reduz a expectativa de inflação futura pelo canal clássico da taxa de juros, essa redução da expectativa de inflação faz com que a taxa real de juros suba, reduzindo o hiato do produto, o qual pode se traduzir em uma queda da inflação. Já um choque cambial, a curva IS apresenta resultados próximos ao da curva de Phillips, embora com uma magnitude maior. No caso de um aumento nos gastos do governo, a expectativa de inflação não gera um efeito significativo sobre a necessidade de financiamento do governo, apenas nos meses iniciais. Assim, um impacto indireto das expectativa de inflação sobre a demanda agregada pode refletir em maiores efeitos inflacionários. 


\subsection{Função de Reação do Banco Central}

Os resultados expostos nessa subseção têm por base perceber como a política monetária via taxa de juros é afetada pela movimentação do desvio da expectativa em relação à meta, na busca de identificar o impacto as flutuações da expectativa dos agentes na taxa de juros.

Esses resultados são apresentados na figura 3 e estabelecem a resposta da Selic, do hiato do produto e do câmbio relacionado ao choque do Desvio das Expectativa (DESVIO). Assim, um aumento da expectativa em relação à meta gera um crescimento da taxa de juros Selic de $0,15 \%$ no primeiro mês, após o terceiro mês a taxa de juros Selic começa a apresentar uma tendência de queda, embora de maneira gradual, propagando-se até o décimo mês, indicando certa inércia da taxa de juros.

Figura 3: Respostas da Função de Reação do Banco Central à Choques de Expectativas
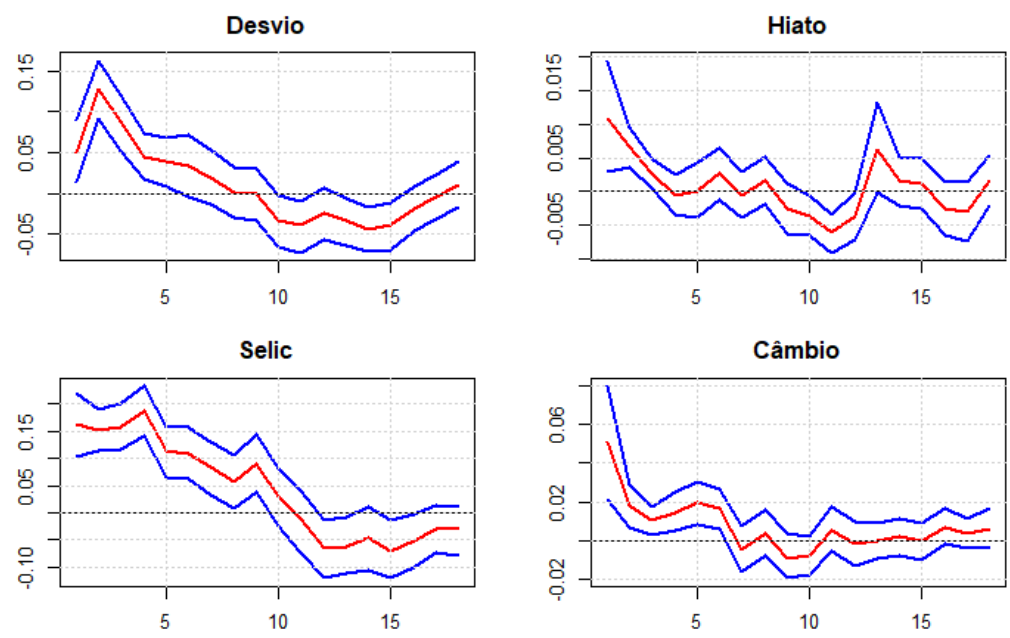

Fonte: Elaborado pelo Autor.

Assim, quando o valor esperado da inflação fica acima da meta de inflação estabelecida para o período, o Banco Central eleva a taxa de juros, visando reprimir a demanda agregada pelo aumento da taxa de juros real, o que pode conduzir a uma redução da inflação, afetando posteriormente a própria expectativa de inflação. É importante destacar que, uma vez que as expectativas se tornam ancoradas na meta, é de se esperar uma redução da taxa de juros de longo prazo via canal da expectativa. Assim, esse resultado sugere que um aumento na taxa Selic tem a tendência a se propagar na economia por um determinado tempo, levando a um maior esforço, por parte de Banco Central, em redirecionar essa variável no futuro.

Além disso, a figura 3 apresenta a resposta do câmbio e do hiato do produto a um choque do desvio das expectativa em relação à média. Observa-se que o impacto no hiato do produto é positivo, embora em uma proporção menor do que o da taxa Selic. O câmbio, por sua vez, apresenta um processo de desvalorização tal como na curva de Phillips. 


\section{Conclusão}

O trabalho objetivou identificar a influência das flutuações nas expectativas de inflação sobre o Regime de Metas de Inflação (RMI), em especial sobre o Índice de Preços ao Consumidor Amplo (IPCA) no Brasil, no período de janeiro 2001 a setembro 2017.

Nesse contexto, o presente estudo demonstrou sucintamente a estrutura do novo consenso macroeconômico, no qual está baseado o Regime de Metas do Brasil, para explicitar a relevância que as expectativas têm em sobre a inflação. No mais, o trabalho apresentou os canais de transmissão da política monetária, tendo como foco o canal das expectativas, para entender a interrelação que a atuação do Banco Central tem, via expectativa, sobre as nuances na demanda agregada que, por sua vez, interfere na inflação.

Nesse contexto, o trabalho se diferencia dos demais que buscaram estimar as curvas de RMI com o intuito de verificar a influência das expectativas tanto diretamente na inflação, tal como se apresenta na curva de Phillips, como de forma indireta, pelo impactado da expectativa na demanda agregada. No mais, a expectativa nesse trabalho é tratada como um canal pelo qual são transmitidas as mudanças nas variáveis do RMI sobre a inflação.

Assim, na curva de Philips verificou-se que a expectativa de inflação impacta de maneira positiva a inflação brasileira desde o primeiro mês, logo quando os agentes precificam um aumento da inflação futura, esse efeito contribui para o aumento da própria inflação no Brasil. Todavia, são os choques cambiais e os choques de oferta que contribuem de maneira mais significativa para as mudanças da inflação brasileira, o que dificulta a capacidade do Banco Central de estabilizar a inflação.

A análise da curva IS demonstrou que a demanda agregada não é impactada fortemente pela expectativa de inflação. Logo, a perspectiva dos agentes em relação à inflação futura não causa grandes mudanças na demanda agregada, embora as nuances do hiato do produto interfiram diretamente na formação de expectativa de inflação. No mais, verificou-se que um aumento da expectativa de inflação faz com que a taxa de juros Swaps DI tenha uma tendência de crescimento, embora passe por um primeiro período de instabilidade.

Por fim, na curva de reação do Banco Central foi possível averiguar que a política monetária realizada pelo Banco Central se torna restritiva quando o desvio da expectativa em torno da meta torna-se positivo, evidenciando a preocupação do Banco Central frente à expectativa de inflação.

Com isso, fazendo uma análise conjunta de todo o regime de metas de inflação, pode-se concluir que a inflação brasileira é influenciada pela percepção dos agentes em relação à própria inflação futura. A formação da expectativa futura de inflação é, pois, influenciada por um conjunto de variáveis que impactam a inflação de maneiras diferentes tanto em magnitude quando em tempo de duração. Nesse ambiente, a autoridade monetária busca conter a expectativa de inflação por meio da taxa de juros que, por sua vez, interfere na demanda agregada, uma vez que a taxa de juros é o principal instrumento de política monetária. Todavia, em comparação com as demais variáveis da curva de Phillips, as mudanças no hiato do produto não provocam grandes mudanças na inflação brasileira, embora impactem na expectativa de inflação. Esse aspecto corrobora a possibilidade de que a inflação seja determinada, em uma maior proporção, pelas variáveis ligadas à oferta da economia e não da 
demanda, cabendo ao Banco Central reduzir apenas os efeitos de propagação dos choques via convergência das expectativas em torno da meta.

\section{Referências Bibliográficas}

Andrade, J. P. \& Divino, J. A. C. (2001), 'Optimal rules for monetary policy in Brazil'.

Areosa, W. (2004), 'Dinâmica da inflação no Brasil: o caso de uma pequena economia aberta', Rio de Janeiro: Departamento de Economia, PUC-Rio .

Arestis, P. \& Sawyer, M. (2008), 'New consensus macroeconomics and inflation targeting: Keynesian critique', Economia e Sociedade 17(SPE), 629-653.

Bevilaqua, A. S., Mesquita, M., Minella, A. et al. (2008), Brazil: taming inflation expectations, in 'Participants in the meeting', p. 139.

Bogdanski, J., Tombini, A. A. \& Werlang, S. R. C. (2000), 'Implementing inflation targeting in Brazil', Werlang, Sergio R., Implementing Inflation Targeting in Brazil (July 2000). Banco Central do Brasil Working Paper (1).

Bonomo, M. A. \& Brito, R. D. (2002), 'Regras monetárias e dinâmica macroeconômica no Brasil: uma abordagem de expectativas racionais', Revista Brasileira de Economia 56(4), 551-589.

Carneiro, D. D., Monteiro, A. M. \& Wu, T. Y. H. (2002), Mecanismos nãolineares de repasse cambial para o IPCA, Technical report, Texto para discussão.

Carrara, A. F. (2016), Choques de oferta e política monetária na economia brasileira: Uma análise do impacto dos preços das commodities na inflação entre 2002 e 2014, PhD thesis, Universidade de São Paulo.

Carroll, C. D. (2003), 'Macroeconomic expectations of households and professional forecasters', the Quarterly Journal of economics 118(1), 269-298.

Clarida, R., Gali, J. \& Gertler, M. (1999), 'The science of monetary policy: a new keynesian perspective', Journal of economic literature 37(4), 1661-1707.

Correa, A. \& Minella, A. (2005), Mecanismos não-lineares de repasse cambial: Um modelo de curva de Phillips com threshold para o Brasil, Technical report, ANPEC-Associação Nacional dos Centros de Pós-Graduação em Economia.

Dezordi, L., D’Agostini, L., Bittencourt, M. \& Curado, M. (2009), 'Regime de metas de inflação para a condução da política monetária brasileira: uma aplicação do modelo VAR', Política Monetária, Bancos Centrais e Metas de Inflação: teoria e experiência brasileira, Rio de Janeiro: Editora FGV, pp. 121-147.

Freitas, P. S., Muinhos, M. K. et al. (2001), A simple model for inflation targeting in Brazil, Banco Central do Brasil.

Mendonça, H. F., Dezordi, L. L. \& Curado, M. L. (2005), 'A determinação da taxa de juros em uma economia sob metas para inflação: o caso brasileiro', Indicadores Econômicos FEE 33(3), 97-110. 
Minella, A., Springer Freitas, P., Goldfajn, I. \& Kfoury Muinhos, M. (2002), 'Inflation targeting in Brazil: lessons and challenges', Banco Central do Brasil Working Paper (53).

Rubio-Ramirez, J. F., Waggoner, D. F. \& Zha, T. (2010), 'Structural vector autoregressions: Theory of identification and algorithms for inference', The Review of Economic Studies 77(2), 665-696.

Santos, F. S. \& Holland, M. (2008), Estimando a demanda agregada no Brasil: o papel dos fatores externos, Technical report, ANPEC-Associação Nacional dos Centros de Pós-Graduação em Economia.

Schettini, B. P., Gouvea, R. R. \& Sachsida, A. (2012), 'Inflação, desemprego e choques cambiais: Estimativas var para a economia brasileira'.

Soares, J. J. S. \& Barbosa, F. H. (2006), 'Regra de Taylor no Brasil: 1999-2005', Encontro Nacional de Economia 34.

Souza Júnior, J. R. C C. \& Caetano, S. M. (2014), 'Regra de Taylor, inércia na política monetária e influência do hiato do produto’.

Vicente, J. V. M. \& Graminho, F. M. (2015), 'Decompondo a inflação implícita', Revista Brasileira de Economia 69(2), 263-284.

Woodford, M. (2009), 'Convergence in macroeconomics: elements of the new synthesis', American economic journal: macroeconomics 1(1), 267-79. 
